\title{
A cross-sectional study of the nasal and fecal microbiota of sows from different health status within six commercial swine farms
}

\author{
Andreia G Arruda ${ }^{\text {Corresp., } 1}{ }^{\text {, Loic Deblais }}{ }^{1}$, Vanessa Hale ${ }^{1}$, Christopher Madden ${ }^{1}$, Monique Pairis-Garcia ${ }^{2}$, Vishal \\ Srivastava $^{1}$, Dipak Kathayat ${ }^{1}$, Anand Kumar ${ }^{3}$, Gireesh Rajashekara ${ }^{1}$ \\ ${ }^{1}$ Department of Veterinary Preventive Medicine, The Ohio State University, Columbus, $\mathrm{OH}$, United States of America \\ 2 Department of Population Health and Pathobiology, North Carolina State University, Raleigh, NC, United States of America \\ 3 Biosecurity and Public Health Group, Bioscience Division, Los Alamos National Laboratory, Los Alamos, NM, United States of America \\ Corresponding Author: Andreia G Arruda \\ Email address: arruda.13@osu.edu
}

Background. Cull sows represent a unique population on swine farms, often representing poor producing or compromised animals, and even though recent studies have reported that the microbiome is associated with susceptibility to diseases, the microbiome in the cull sow population has not been explored. The main objective of this study was to investigate whether there were differences in fecal and upper respiratory tract microbiota composition for groups of sows of different health status (healthy, cull, and compromised/ clinical sows) and from different farms (1 to 6). Methods. Six swine farms were visited once. Thirty individual fecal samples and nasal swabs were obtained at each farm and pooled by five across health status and farm. Samples underwent 16S rRNA gene amplicon sequencing and nasal and fecal microbiota were analyzed using QIIME2 v.2021.4. Results. Overall, the diversity of the nasal microbiota was lower than the fecal microbiota ( $p<$ 0.01 ). No significant differences were found in fecal or nasal alpha diversity by sow's health status or by farm. There were significant differences in nasal microbial composition by farm and health status (PERMANOVA, $p<0.05$ ), and in fecal microbiota by farm (PERMANOVA, $p<0.05$ ), but not by health status. Lastly, at the L7 level, there was one differentially abundant taxa across farms for each nasal and fecal pooled samples.

Discussion. This study provided baseline information for nasal and fecal microbiota of sows under field conditions, and results suggest that farm of origin can affect microbial diversity and composition. Furthermore, sow's health status may have an impact on the nasal microbiota composition. 
1 A cross-sectional study of the nasal and fecal

2 microbiota of sows from different health status within

3 six commercial swine farms

4

5

6

7

8

Andréia Gonçalves Arruda ${ }^{1 *}$, Loic Deblais ${ }^{1}$, Vanessa Hale ${ }^{1}$, Christopher Madden ${ }^{1}$, Monique

Pairis-Garcia ${ }^{2}$, Vishal Srivastava ${ }^{1}$, Dipak Kathayat ${ }^{1}$, Anand Kumar ${ }^{3}$, Gireesh Rajashekara ${ }^{1}$

${ }^{1}$ Department of Veterinary Preventive Medicine, The Ohio State University, Columbus, Ohio, United States of America

${ }^{2}$ Department of Population Health and Pathobiology, North Carolina State University, Raleigh, North Carolina, United States of America

${ }^{3}$ Biosecurity and Public Health Group, Bioscience Division, Los Alamos National Laboratory, New Mexico, United States of America

Corresponding Author:

Andreia Arruda ${ }^{1}$

1920 Coffey Road, Columbus, OH, 43215, USA

Email address: arruda.13@osu.edu

\section{Abstract}

Background. Cull sows represent a unique population on swine farms, often representing poor producing or compromised animals, and even though recent studies have reported that the microbiome is associated with susceptibility to diseases, the microbiome in the cull sow population has not been explored. The main objective of this study was to investigate whether there were differences in fecal and upper respiratory tract microbiota composition for groups of sows of different health status (healthy, cull, and compromised/ clinical sows) and from different farms (1 to 6).

Methods. Six swine farms were visited once. Thirty individual fecal samples and nasal swabs were obtained at each farm and pooled by five across health status and farm. Samples underwent 16S rRNA gene amplicon sequencing and nasal and fecal microbiota were analyzed using QIIME2 v.2021.4.

Results. Overall, the diversity of the nasal microbiota was lower than the fecal microbiota $(\mathrm{p}<$ 0.01 ). No significant differences were found in fecal or nasal alpha diversity by sow's health status or by farm. There were significant differences in nasal microbial composition by farm and health status (PERMANOVA, $\mathrm{p}<0.05$ ), and in fecal microbiota by farm (PERMANOVA, $\mathrm{p}<$ 0.05 ), but not by health status. Lastly, at the L7 level, there was one differentially abundant taxa across farms for each nasal and fecal pooled samples.

Discussion. This study provided baseline information for nasal and fecal microbiota of sows under field conditions, and results suggest that farm of origin can affect microbial diversity and 
42 composition. Furthermore, sow's health status may have an impact on the nasal microbiota

43 composition.

\section{Introduction}

Culling refers to the process of removing animals from a breeding herd in order to optimize productivity and profitability. This is an essential and common practice conducted on commercial swine herds and it is typical for large systems to cull up to $50 \%$ of the herd on an annual basis (D’Allaire, Stein \& Leman, 1987; Stein et al., 1990; Stalder et al., 2003). Sows, which refer to reproductively mature females, are normally culled for reasons that include but are not limited to low production efficiency, age, poor reproductive traits, and/ or presumed compromised immune status. However, the definitive underlying reason for culling is often not completely elucidated or recorded. In addition, culls sows may remain on the farm, mixed with the general population, for a significant time period as transportation of mature pigs is logistically challenging. This results in the maintenance of a consistent subpopulation of potentially compromised animals that may serve as a source of pathogens to the general population of healthy sows. Even though recent work has focused on the role of cull sows on disease transmission outside of the farm (i.e. auctions, sale yards etc.) (Sutherland, 2018), little to no work has been devoted to assessing the role of cull sows in the maintenance and re-emergence of pathogens and disease on-farm.

62

The microbiome is defined as the microbial community within or on a body site and is known to play an important role in human and animal health (Round \& Mazmanian, 2009; Young, 2017; Nowland et al., 2019) and animal production (Jami, White \& Mizrahi, 2014). The study of the swine microbiome and its impact in respiratory and systemic diseases has been an emerging area of study within the past few years (Niederwerder, 2017). More specifically, published papers have focused on investigating the associations between the piglet gut or fecal microbiome and intestinal immunity development (Schokker et al., 2014), and performance upon the presence of pathogenic viruses and their co-infections (Niederwerder et al., 2016; Ober et al., 2017). A recent paper has reviewed several publications on piglets' intestinal microbiome and its association with post weaning diarrhea, with a focus on antimicrobial use (Gresse et al., 2017). A few other studies have also described the associations between the piglet nasal microbiota and presence of pathogenic bacteria such as Staphylococcus sp and Haemophilus parasusis (Weese et al., 2014; Espinosa-Gongora et al., 2016)

Previous work has explored the impact of health status and geographic location on swine microbiota (Correa-Fiz, Fraile \& Aragon, 2016); in addition, recent work conducted under laboratory and semi-experimental conditions has determined that the gut and nasal microbiota are associated with susceptibility to respiratory conditions such as porcine respiratory and reproductive syndrome (PRRS) (Niederwerder et al., 2016), porcine circovirus type 2 associated disease (Niederwerder et al., 2016), and Glasser's disease (Correa-Fiz, Fraile \& Aragon, 2016). 
83 However, the vast majority of the studies published in the literature focused on piglet

84 populations and did not investigate the effect of health status or farm of origin on the microbiota 85 of sows.

86

87 Therefore, the objectives of this study were to:

88 1) Characterize baseline fecal and nasal microbiota of adult sows under field conditions,

89 2) Investigate the association between health status (including healthy, cull, and compromised/

90 clinical sows) and nasal and fecal microbial composition and diversity. For the purpose of this

91 study, cull sows were defined as an adult female that was identified to be removed from the herd;

92 and compromised or clinical sows were defined as sows presenting acute clinical signs of disease

93 that could be visually assessed by the attending veterinarian.

94 3) Assess the association between farm of origin and nasal and fecal microbial composition and 95 diversity.

\section{Materials \& Methods}

\section{Study population and sampling protocol}

This study has been approved by The Ohio State University's Institutional Animal Care and Use Committee (IACUC Protocol \#2017A00000060), as well as by the Institutional Biosafety Committee (IBC Protocol \#2017R00000041).

A cross-sectional observational study was conducted during July of 2017. The project included enrollment of sows distributed within six privately owned commercial farrow-to-wean swine farms from the same production company located in the U.S. Mideast region. A commercial farm was defined as a swine farm that raised pigs with the intent to commercialize them (in this case of farrow-to-wean farms, the farms were producing and selling weaned piglets to downstream farms that would raise them to market). All sows were fed commercial diets from the same source that met the Nutrient Requirements of Swine (National Research Council,

112 2012). Basic farm descriptors are shown in Table 1. Healthy sows were defined as an adult

113 female with no evidence of clinical signs of disease at the time of data collection. Healthy sows

114 were included regardless of stage of production (i.e. pregnant, lactating or dry). Cull sows were

115 defined as adult females that was identified to be removed from the herd based on the standard

116 operating procedure implemented by the farm staff. Investigators were using sow cards (paper

117 records) in order to identify culled sows. The primary reasons for animals to be culled was if

118 they did not achieve acceptable reproductive performance. Compromised or clinical sows (the

119 two terms are used interchangeably throughout the manuscript) were defined as sows presenting acute clinical signs of disease that could be visually assessed by the attending veterinarian. This included the following categories: lameness, severe shoulder ulcers, active abortion, and poor body condition score. Each farm contributed an equal number of sampled sows for each health 
123 status examined herein (healthy, cull and compromised / clinical); with 10 sows sampled for

124 each health status in each farm.

125

126 Farms were visited once during the month of July of 2017 for collection of fecal samples and nasal swabs from all sows within a farm on the same day. Fecal samples were used in this study as a non-invasive way to examine the gut microbiota. A total of 30 fecal samples and 30 nasal swabs were obtained per farm (ten for each type of animal) for a total of 360 samples (180 fecal samples and 180 nasal swabs). A schematic of sampling design is presented in Figure 1, along with relative geographical location for the sampled farms.

132

For nasal swab sampling, pigs were restrained using a snare and the swab (Puritan ${ }^{\mathrm{TM}}$ PurFlock ${ }^{\mathrm{TM}}$ Ultra Sterile Flocked Swabs) was inserted into the ventral passage of each nostril. Nasal samples were collected for three seconds (per nasal passage) and placed in a dry tube. Following this, a fecal sample was collected using digital palpation of the rectum to remove feces with a sterile glove. Fecal samples were then placed into sterile bags.

138

Sample pooling, genomic DNA extraction and sequencing

Fecal and nasal swabs were collected and placed in tubes on dry ice immediately after collection. Samples were then transported to the Food Animal Health Research Program (The Ohio State University) laboratory in Wooster, Ohio, and transferred into a $-80 \mathrm{C}$ freezer within 24 hours where they remained until extraction. For nasal swabs, two milliliters of phosphate-buffered saline (PBS) were added to each nasal swab tube, tubes were vortexed for $30 \mathrm{sec}$. The samples were divided into 2 subsamples: one $1 \mathrm{ml}$ sample was stored at $-80 \mathrm{C}$ for future use and the other $1 \mathrm{ml}$ sample was centrifuged at $13,000 \mathrm{~g}$ for $15 \mathrm{~min}$. The resulting pellet was resuspended in 250 $\mu 1$ of PBS and used for DNA extraction. Fecal and nasal swab samples were pooled across 5 animals of the same status by farm; so each farm contributed two pools of each health status containing samples of five animals each, as shown in Figure 1. The samples selected to be part of the same pool were randomly selected, and the nasal and fecal pool sets were composed of the same animals. The pools consisted of $0.5 \pm 0.015 \mathrm{~g}$ of feces per sample and $45 \mu \mathrm{l}$ of nasal swab suspension per sample. There were 72 pooled samples in total (36 fecal and 36 nasal swab pooled samples) and the pooled sample was considered our unit of analysis. Genomic DNA extraction of the pooled fecal $(0.5 \pm 0.015 \mathrm{~g})$ or nasal $(225 \mu \mathrm{l})$ swab samples was conducted using the PureLink Microbiome DNA Purification Kit (Life Technologies, Invitrogen Corp.) followed by RNAse treatment (10 units for 1 hour), as previously described (Deblais et al., 2018). Four blank (empty) extractions were also performed as negative controls and sequenced along with the rest of the samples. It is important to note that this commercial kit lyses the cells using chemical (lysis buffer + enhancer [guanidine isothiocyanate]), thermic $\left(65^{\circ} \mathrm{C}\right.$ for 10 minutes), and mechanical (bead beating at full speed for 10 minutes) approaches. Next 
162 generation sequencing library preparations and Illumina MiSeq sequencing were conducted at

163

164

165

166

167

168

169

170

171

172

173

174

175

176

177

178

179

180

181

182

183

184

185

186

187

188

189

190

191

192

193

194

195

196

197

198

199

200

201

202

203

204

205

the Bioscience Division, Los Alamos National Laboratory, NM.

Briefly, DNA samples were quantified using a Qubit 2.0 Fluorometer (Invitrogen, Carlsbad, CA) and DNA quality was checked on a $0.6 \%$ agarose gel using E-gel electrophoresis system (ThermoFischer Scientific, Grand Island, NY). A total of $50 \mathrm{ng}$ DNA was used to generate amplicons of the V3-V4 hypervariable regions of the bacterial and Archaeal 16S rRNA gene using primers $341 \mathrm{~F}$ and 806R. The first round of PCR amplified the V3-V4 region using KAPA HIFI HotStart Ready Mix (Kapa Biosystems, Wilmington, MA) with following PCR conditions; $95^{\circ} \mathrm{C}$ for 3 minutes, 20 cycles of $95^{\circ} \mathrm{C}$ for 30 seconds, $55^{\circ} \mathrm{C}$ for 30 seconds and $72^{\circ} \mathrm{C}$ for 30 seconds and an extension of $72^{\circ} \mathrm{C}$ for 5 minutes. The second round of PCR added the Illumina specific sequencing adapter sequences and unique indexes using the Nextera XT Index Kit v2 (Illumina, San Diego, CA) and KAPA HIFI HotStart Ready Mix (Kapa Biosystems, Wilmington, MA) with following PCR conditions; $95^{\circ} \mathrm{C}$ for 3 minutes, 8 cycles of $95^{\circ} \mathrm{C}$ for $30 \mathrm{~seconds}, 55^{\circ} \mathrm{C}$ for 30 seconds and $72^{\circ} \mathrm{C}$ for 30 seconds and an extension of $72^{\circ} \mathrm{C}$ for 5 minutes. The amplicons were cleaned up using AMPure XP beads (Beckman Coulter, Indianapolis, IN). The concentration of the amplicons pool was obtained using the Qubit dsDNA HS Assay (ThermoFisher Scientific, Grand Island, NY). The average size of the library was determined by the Agilent High Sensitivity DNA Kit (Agilent, Santa Clara, CA). An accurate library quantification was determined using the Library Quantification Kit - Illumina/Universal Kit (KAPA Biosystems, Wilmington, MA). The amplicon pool was sequenced on the Illumina MiSeq generating paired end $300 \mathrm{bp}$ reads. The amplicon pool was demultiplexed using Illumina's bcl2fastq. Sequencing data are publicly available at NCBI SRA: PRJNA674564.

\section{Taxonomic microbiota analyses and statistical analyses}

Raw, single-end sequence reads were processed using QIIME2 v. 2021.4 (Bolyen et al., 2019). The DADA2 plugin was used to truncate reads at $280 \mathrm{bp}$ and to denoise the single-end reads (Callahan et al., 2016). We opted for single end reads over paired end due to the poor quality of the $\mathrm{R} 2$ reads. Taxonomy was assigned in QIIME2 using SILVA version 138.1, with a 99\% similarity threshold using the full length 16S rRNA gene classifier (Quast et al., 2013; Yilmaz et al., 2014). All fecal samples with fewer than 5600 reads and all nasal samples with fewer than 500 reads were removed from analyses. To compare alpha diversity between nasal and fecal samples, all samples were rarefied at 500 reads. We also rarefied at 500 reads to compare nasal samples by farm and health status. To compare fecal samples by farm and health status, we rarified at 5600 reads. In total, we retained all fecal samples $(n=36)$ and 19 out of 36 nasal samples for further analysis. To assess the effects of rarefaction, we also analyzed fecal and nasal samples without rarefaction (excluding fecal samples with fewer than 5600 reads and nasal samples with fewer than 500 reads). We also analyzed nasal samples (rarefied and non-rarefied) excluding samples with fewer than 1000 reads.

Alpha diversity was analyzed using Shannon and Faith's Phylogenetic Diversity (PD) indices. A Kruskal-Wallis test was used to compare alpha diversity and read numbers by health status and farm. An adjusted P-value lower than 0.05 was considered significant.

PeerJ reviewing PDF | (2020:10:54076:4:0:NEW 5 Aug 2021) 
206 Beta diversity indices were compared using permutational multivariate analysis of variances

207 (PERMANOVA) and permutational analysis of multivariate dispersions (PERMDISP) between

208 Bray Curtis distance matrices. P-values were corrected for multiple comparisons using the

209 Benjamini-Hochberg FDR correction, and values less than 0.05 were considered significant.

210 PERMANOVAs were performed on the fecal and nasal samples by farm and by health status. An

211 analysis of composition of microbes (ANCOM) was used to identify differentially abundant

212 nasal and fecal taxa by farm and health status. An ANCOM performs pairwise comparisons of

213 microbial species ratios and generates a $W$-value, which indicates how many times a null

214 hypothesis is rejected across comparisons. The maximum $\mathrm{W}$ value in any data set is limited by

215 the total number of microbial taxa within that data set. For all samples, we filtered out taxa that

216 had fewer than 10 reads and occurred in fewer than two samples prior to ANCOM analysis. No

217 additional filtering was performed on nasal samples due to the low microbial diversity in the

218 majority of samples. ANCOMs were performed at the L7 level, which is roughly equivalent to a

219

220

221

222

223

224

225

226

227

228

229

230

231

232 species level; although deeper genome sequencing is necessary for true species differentiation.

\section{Results}

One hundred and eighty animals were sampled across the six farms; comprising of a total of 60 healthy sows, 60 cull sows, and 60 compromised / clinical sows. Reasons for sows to be in the 'compromised' or 'clinical' group included low body condition score (48.3\%), acute abortion $(23.3 \%)$ and shoulder ulcer (15.0\%). For the 'cull' group, the main reason for culling was low performance (which included low number of weaned pigs, high pre-weaning mortality, and low number of born alive piglets; $50.0 \%$ ), unknown (missing information on the sow card; $28.3 \%$ ), and return to estrus $(10.0 \%)$. A breakdown for most common culling reasons by farm is provided on Table 1. The mean parity for compromised/ clinicalanimals was $2.9(\mathrm{SD}=2.0)$; for cull sows, 5.2 (1.9), and for heathy sows, 3.0 (2.1). A breakdown for parity distribution by farm is provided on Table 1.

233

234

\section{$16 S$ rRNA Gene Amplicon Sequencing}

235 We obtained a total of 1,759,761 reads with a mean read length of 294 base pairs (Supplemental

236 Table 1). Of the four negative controls, only one yielded any reads (3 reads total), and all 3 reads

237 were identified as chloroplasts. Chloroplasts, mitochondria, and eukaryotic reads were then

238 removed bioinformatically prior to further analyses. A total of 493 taxa were identified across all

239 samples. The mean number of reads per nasal swab pool $(n=36)$ was 2,572 ; the median was

240 914.5, with a range of 1-27,870 reads (Supplemental Table 2). Nasal samples with fewer than

241500 reads $(\mathrm{n}=17)$ were excluded from analysis. For the 36 pooled fecal samples, we obtained a

242 total of 901,440 reads. The mean number of reads per fecal pooled sample was 25,040 , and the

243 median was 22,256, with a range of 5,694-62,677 reads (Supplemental Table 3). All fecal

244 samples were included in our analyses, which were performed rareified at 5600 reads and 
245 unrarified. To facilitate a direct comparison between nasal and fecal samples, we also performed

246 a separate analysis with fecal and nasal samples together rarefied at 500 reads and unrarefied.

247

248

Alpha diversity results

249

Overall, nasal samples harbored a significantly lower microbial diversity compared to the fecal

250 samples (Faith's PD, $p<0.001$; Shannon, $p<0.001$; Supplemental Figure 1a). No significant

251 differences in fecal or nasal diversity (Shannon index) were detected by health status (healthy,

252 cull and compromised / clinical) or by farm (Kruskal-Wallis, all $p>0.05$, Figure 2, Supplemental

253 Figure 1b, Supplemental Table 4).

254

255

256

Beta diversity results

257 There were significant differences in overall nasal microbial composition by farm (Bray-Curtils PERMANOVA, pseudo-F $=1.83, \mathrm{p}=0.002$, Figure 3a, Supplementary Figure 2a, Supplemental

259 Table 4); although, no pairwise comparisons were significant. Overall nasal microbial composition by health status also differed significantly (PERMANOVA, pseudo-F $=1.64, \mathrm{p}=$ 0.017 , Figure 3b, Supplementary Figure $2 b$ ) with healthy versus cull as the only significant pairwise comparison $(p=0.033)$. These differences were not explained by dispersion (PERMDISP by farm: pseudo-F $=0.577, \mathrm{p}=0.795$; by health status: pseudo- $\mathrm{F}=2.11, \mathrm{p}=0.148$ ) or number of reads by farm or health status (Kruskal-Wallis by farm: $p=0.36$; by health status: $p$ $=0.28$ ). When we examined the pooled fecal microbiota of sows, we found significant differences in overall microbial composition by farm (PERMANOVA, pseudo-F $=3.16, p=$ 0.001, Figure 4a, Supplementary Figure 3a, Supplemental Table 4) with significant pairwise differences between all farms $(\mathrm{p}<0.05)$ with the exception of farms 4 and $5(\mathrm{p}=0.08)$ and farms 5 and $6(p=0.1)$. No significant differences in pooled fecal microbial composition were found based on health status ( $p=0.663$, Figure $4 b$, Supplementary Figure $3 b)$.

270

271

In terms of relative abundances, the most abundant phyla in the pooled nasal samples was

272

273 Proteobacteria, followed by Firmicutes, Actinobacteria and Bacteroidetes (Figure 5a). The most abundant nasal genera across all pooled samples was Moraxella. At the L7 level, we identified one differentially abundant nasal taxa by farm: a taxa in the Micrococcaeae family, genera Nesterenkonia (ANCOM, W =32), which was only present on farm 6 (Supplemental Table 5). No nasal taxa were differentially abundant by health status. In terms of relative abundance in the pooled fecal samples, Firmicutes was the most abundant phyla, followed by Bacteroidetes, Euryarchaeotes, Spirochetes, Actinobacteria and Proteobacteria (Figure 5b). Within the

279 Firmicutes phyla, the most abundant family was Lachnospiraceae, and the most abundant genera across all fecal samples were Lactobacillus and Methanobrevibacter (an Archaea). At the L7 (roughly species) level in fecal samples, we identified one differentially abundant taxa by farm: it was a taxa in the order Bacteroidales, genera F082 (ANCOM, W = 140), which was broadly present at low abundances across all samples from farms 1, 4, 5 and 6 but almost entirely absent 
284 on farms 2 and 3 (Supplemental Table 6). No fecal taxa were differentially abundant by health

285 status.

286

287

288

289

290

291

292

293

294

295

296

297

298

299

300

301

302

303

304

305

306

307

308

309

310

311

312

313

314

315

\section{Discussion}

Cull sows are important from both a disease transmission and welfare perspective but are an understudied subpopulation of animals within commercial swine farms. Studies looking at both fecal and nasal microbiota of adult female pigs are lacking in the literature. Therefore, the main objective of this study was to characterize nasal and fecal microbiota of sows that differed by health status and farm of origin under field conditions.

Our study did not show statistical differences in nasal microbial alpha diversity by health status, or by farm. Few studies have examined swine nasal microbial diversity and reports vary: one study reports significantly decreased diversity linked to Glasser's disease (Correa-Fiz, Fraile \& Aragon, 2016) while another reports no change in diversity linked to methicillin-resistant Staphylococcus aureus (MRSA) carriage (Weese et al., 2014). A few studies have also reported changes in nasal microbiota upon use of antimicrobial treatments, all conducted in young animals (which are different from our population of interest). A study conducted in Spain reported the use of peri-natal treatment with antimicrobials (including penicillin, streptomycin and tulathromycin in one farm, and ceftiofur and tulathromycin in a second farm) in 3-4 week old piglets to be associated with a decrease in nasal microbiota diversity as compared to a period without using these peri-natal treatments. (Correa-Fiz et al., 2019). Likewise, a study conducted in the US showed that oxytetracycline administration reduced nasal microbiota diversity in 3week-old piglets (Mou et al., 2019). In this study, on-farm antimicrobial treatments were not available to investigators, which did not allow for further investigations. Additionally, the only disease issue reported amongst all six farms in the month prior to sampling was a recent swine influenza outbreak on farm 1. Despite this, we observed no differences in microbial diversity by farm or health status. All farms have reported to not have had outbreaks for the main pathogens of importance in swine including porcine reproductive and respiratory syndrome, Mycoplasma $s p$. and porcine epidemic diarrhea.

318 size (number of sows) and parity distribution are higher for farm 5, which could have been

319 contributors for the observed trends in microbiota composition.

320

321

322

In terms of relative abundance in nasal swab samples, Proteobacteria was the largest represented phyla, followed by Firmicutes, Actinobacteria and Bacteroidetes. These results are similar to

323 swine-related nasal microbiota studies conducted in Canada, the United Kingdom and Spain 
324 (Weese et al., 2014; Slifierz, Friendship \& Weese, 2015; Correa-Fiz, Fraile \& Aragon, 2016)

325

326

327

328

329

330

331

332

333

334

335

336

337

338

339

340

341

342

343

344

345

346

347

348

349

350

351

352

353

354

355

356

357

358

359

360

361

362

363

which report Proteobacteria as the most abundant phyla, followed by a large abundance in Bacteroidetes, and Firmicutes. In contrast, the abundance of Actinobacteria in our study is higher than what was previously reported (Weese et al., 2014; Correa-Fiz, Fraile \& Aragon, 2016), and the abundance of Tenericutes (particularly Mycoplasmacetae family), phyla which has been recently reclassified into the Firmicutes phylum (Parks et al., 2018), was more prominent in the European study (Correa-Fiz, Fraile \& Aragon, 2016) compared to our current study. These differences could be related to animal age given previous studies were conducted with piglets or slaughter animals [approximately six months of age], which are younger than our adult female study population (1-3 years of age). Nesterenkonia was the only taxa identified as differentially abundant by farm, and was only found on farm 6 . The relevance of this taxa in swine is unclear; although it has been identified previously in studies on the human lower repiratory tract and gut microbiota (Chander et al., 2017; Li et al., 2019).

Most of the shared taxa for fecal samples in our study were in the Firmicutes and Bacteroidetes families. This agrees with a meta-analysis published in 2017, which reported that those two phyla accounted for nearly $85 \%$ of the $16 \mathrm{~S}$ rRNA gene amplicon sequences among over 930 swine gastrointestinal samples (Holman et al., 2017). This meta-analysis also pointed out that the main factors influencing swine gut microbiota were study itself, including technical factors such as extraction and sequencing, gastrointestinal sample location, and animal age, which makes comparisons between studies a challenge. A study by Kim et al. (Kim \& Isaacson, 2015) analyzed fecal samples of various aged pigs (including sows) and reported that the most common microbial families in sows were Prevotellaceae, Ruminococcaceae, Lachnospiraceae, and Streptococcaceae, which were also present across most of the fecal samples in our study. Those authors also reported a large proportion of "unclassified" taxa, which did not occur in our case.

Even though the farms enrolled in the current study were under the same management practice (i.e. herd veterinarian, health and biosecurity protocols, treatment protocols, feed source), there were likely inherent differences regarding past disease challenges, herd productivity, and physical characteristics of the farm (e.g. farm size, number of workers, environment, biosecurity etc.) that were not fully captured or available to investigators in this study, and may have influenced differences observed. For example, farm size and parity distribution were among some of the differences observed between farms that were different in regards to microbial composition metrics (Table 1).

An important limitation of this study includes the pooling of samples (five sows were represented in each pooled sample). This resulted in only two samples per health status per farm, a small sample size that may have limited our statistical power. This pooling further limited our ability to evaluate the relationship between individual-level variables such as parity, age, production stage and reproductive performance on nasal and fecal microbiota. A second

Peer) reviewing PDF | (2020:10:54076:4:0:NEW 5 Aug 2021) 
364 limitation includes the lack of clean/blank swabs extracted as negative controls. Low biomass

365

366

367

368

369

370

371

372

373

374

375

376

377

378

379

380

381

382

383

384

385

386

387

388

389

390

391

392

393

394

395

396

397

398

399

400

401

402

samples, such as some of the nasal samples in this study, are highly subject to contamination, and we cannot rule out the possibility of swab-contaminants in these samples. However, we did include negative controls for extraction (kits) and sequencing, and we analyzed the nasal sample data with and without rarefaction and after removing samples with fewer than 500 or 1000 reads in an attempt to eliminate the samples at greatest risk of contamination. The low number of reads in the nasal samples likely further limited our statistical power and ability to distinguish true differences between groups. Moreover, in the nasal sample analysis, rarefaction at 500 reads is a low sampling depth that could have limited our ability to detect and compare the true level of diversity within these samples. Despite this, when we analyzed nasal samples with and without rarefaction at a minimum threshold of either 500 or 1000 reads, the results were comparable in all 4 analyses (Supplemental Table 4). With all these considerations, the results presented herein should be considered preliminary, and interpreted as an initial baseline for our population (s) of interest.

In addition, we further acknowledge the potential for misclassification bias when selecting study subjects. This stems from the fact that on swine farms, the use of "sow cards", which are paperbased animal records, is a common way of keeping production records and "flagging" animals that need to be culled. In many cases, the reason for culling is not always specified. As such, during farm visits, investigators relied on these cards to identify animals that were to be culled, which were then included in the study. Confirmation with the farm manager or farm personnel was attempted whenever possible to minimize this potential source of bias. Future studies could expand sample size and should focus on exploring the association between the fecal and nasal microbiota and detailed demographic (e.g. age), health (e.g. antimicrobial treatment history), and production parameters at the animal or herd levels.

\section{Conclusions}

In conclusion, this study provided baseline information for nasal and fecal microbiota of sows under field conditions. Our results suggest that farm of origin can affect nasal and fecal microbial diversity and composition, and health status of animals (compromised / clinical, healthy and cull) can affect alpha diversity. Given the study limitations, these analyses should be expanded to a larger number of animals in the future.

\section{Acknowledgements}

The authors would like to acknowledge farm owners, managers and personnel, and undergraduate students for help with sampling collection. This project was partially supported by a Los Alamos National Laboratory (LANL) internal Grant (20170671PRD2) and has been

Peer) reviewing PDF | (2020:10:54076:4:0:NEW 5 Aug 2021) 
403

404

405

406

407

408

409

410

411

412

413

414

415

416

417

418

419

420

421

422

423

424

425

426

427

428

429

430

431

432

433

434

435

436

437

438

439

440

441

442

approved by the LANL Classification Office (DFS-CL) with assigned the number LA-UR-2028622.

\section{References}

Bolyen E, Rideout JR, Dillon MR, Bokulich NA, Abnet CC, Al-Ghalith GA, Alexander H, Alm EJ, Arumugam M, Asnicar F, Bai Y, Bisanz JE, Bittinger K, Brejnrod A, Brislawn CJ, Brown CT, Callahan BJ, Caraballo-Rodríguez AM, Chase J, Cope EK, Da Silva R, Diener C, Dorrestein PC, Douglas GM, Durall DM, Duvallet C, Edwardson CF, Ernst M, Estaki M, Fouquier J, Gauglitz JM, Gibbons SM, Gibson DL, Gonzalez A, Gorlick K, Guo J, Hillmann B, Holmes S, Holste H, Huttenhower C, Huttley GA, Janssen S, Jarmusch AK, Jiang L, Kaehler BD, Kang KB, Keefe CR, Keim P, Kelley ST, Knights D, Koester I, Kosciolek T, Kreps J, Langille MGI, Lee J, Ley R, Liu Y-X, Loftfield E, Lozupone C, Maher M, Marotz C, Martin BD, McDonald D, McIver LJ, Melnik AV, Metcalf JL, Morgan SC, Morton JT, Naimey AT, Navas-Molina JA, Nothias LF, Orchanian SB, Pearson T, Peoples SL, Petras D, Preuss ML, Pruesse E, Rasmussen LB, Rivers A, Robeson MS, Rosenthal P, Segata N, Shaffer M, Shiffer A, Sinha R, Song SJ, Spear JR, Swafford AD, Thompson LR, Torres PJ, Trinh P, Tripathi A, Turnbaugh PJ, Ul-Hasan S, van der Hooft JJJ, Vargas F, Vázquez-Baeza Y, Vogtmann E, von Hippel M, Walters W, Wan Y, Wang M, Warren J, Weber KC, Williamson CHD, Willis AD, Xu ZZ, Zaneveld JR, Zhang Y, Zhu Q, Knight R, Caporaso JG. 2019. Reproducible, interactive, scalable and extensible microbiome data science using QIIME 2. Nature Biotechnology 37:852-857. DOI: 10.1038/s41587-019-0209-9.

Callahan BJ, McMurdie PJ, Rosen MJ, Han AW, Johnson AJA, Holmes SP. 2016. DADA2: High-resolution sample inference from Illumina amplicon data. Nature Methods 13:581583. DOI: $10.1038 /$ nmeth.3869.

Chander AM, Nair RG, Kaur G, Kochhar R, Dhawan DK, Bhadada SK, Mayilraj S. 2017. Genome Insight and Comparative Pathogenomic Analysis of Nesterenkonia jeotgali Strain CD08_7 Isolated from Duodenal Mucosa of Celiac Disease Patient. Frontiers in Microbiology 8:129. DOI: 10.3389/fmicb.2017.00129

Correa-Fiz F, Fraile L, Aragon V. 2016. Piglet nasal microbiota at weaning may influence the development of Glässer's disease during the rearing period. BMC genomics 17:1-14.

Correa-Fiz F, Gonçalves dos Santos JM, Illas F, Aragon V. 2019. Antimicrobial removal on piglets promotes health and higher bacterial diversity in the nasal microbiota. Scientific Reports 9. DOI: 10.1038/s41598-019-43022-y.

D’Allaire S, Stein TE, Leman AD. 1987. Culling patterns in selected Minnesota swine breeding herds. Canadian Journal of Veterinary Research 51:506.

Deblais L, Helmy YA, Kathayat D, Huang H, Miller SA, Rajashekara G. 2018. Novel imidazole and methoxybenzylamine growth inhibitors affecting Salmonella cell envelope integrity and its persistence in chickens. Scientific reports 8:1-17. 
443 Espinosa-Gongora C, Larsen N, Schønning K, Fredholm M, Guardabassi L. 2016. Differential

444

445

446

447

448

449

450

451

452

453

454

455

456

457

458

459

460

461

462

463

464

465

466

467

468

469

470

471

472

473

474

475

476

477

478

479

480

481

482

483 analysis of the nasal microbiome of pig carriers or non-carriers of Staphylococcus aureus. PloS one 11:e0160331.

Gresse R, Chaucheyras-Durand F, Fleury MA, Van de Wiele T, Forano E, Blanquet-Diot S. 2017. Gut microbiota dysbiosis in postweaning piglets: understanding the keys to health. Trends in microbiology 25:851-873.

Holman DB, Brunelle BW, Trachsel J, Allen HK. 2017. Meta-analysis to define a core microbiota in the swine gut. MSystems 2.

Jami E, White BA, Mizrahi I. 2014. Potential role of the bovine rumen microbiome in modulating milk composition and feed efficiency. PloS one 9:e85423.

Kim HB, Isaacson RE. 2015. The pig gut microbial diversity: understanding the pig gut microbial ecology through the next generation high throughput sequencing. Veterinary microbiology 177:242-251.

Li K-J, Chen Z-L, Huang Y, Zhang R, Luan X-Q, Lei T-T, Chen L. Dysbiosis of lower respiratory tract microbiome are associated with inflammation and microbial function variety. 2019. Respiratory Research 20:272. http://doi.org/10.1186/s12931-019-1246-0.

Mou KT, Allen HK, Alt DP, Trachsel J, Hau SJ, Coetzee JF, Holman DB, Kellner S, Loving CL, Brockmeier SL. 2019. Shifts in the nasal microbiota of swine in response to different dosing regimens of oxytetracycline administration. Veterinary Microbiology 237:108386. DOI: 10.1016/j.vetmic.2019.108386.

National Research Council. 2012. Nutrient Requirements of Swine: Eleventh Revised Edition. DOI: $10.17226 / 13298$.

Niederwerder MC. 2017. Role of the microbiome in swine respiratory disease. Veterinary microbiology 209:97-106.

Niederwerder MC, Jaing CJ, Thissen JB, Cino-Ozuna AG, McLoughlin KS, Rowland RR. 2016. Microbiome associations in pigs with the best and worst clinical outcomes following coinfection with porcine reproductive and respiratory syndrome virus (PRRSV) and porcine circovirus type 2 (PCV2). Veterinary microbiology 188:1-11.

Nowland TL, Plush KJ, Barton M, Kirkwood RN. 2019. Development and function of the intestinal microbiome and potential implications for pig production. Animals 9:76.

Ober RA, Thissen JB, Jaing CJ, Cino-Ozuna AG, Rowland RR, Niederwerder MC. 2017. Increased microbiome diversity at the time of infection is associated with improved growth rates of pigs after co-infection with porcine reproductive and respiratory syndrome virus (PRRSV) and porcine circovirus type 2 (PCV2). Veterinary microbiology 208:203-211.

Parks DH, Chuvochina M, Waite DW, Rinke C, Skarshewski A, Chaumeil P-A, Hugenholtz P. 2018. A standardized bacterial taxonomy based on genome phylogeny substantially revises the tree of life. Nature biotechnology 36:996-1004.

Quast C, Pruesse E, Yilmaz P, Gerken J, Schweer T, Yarza P, Peplies J, Glöckner FO. 2013. The SILVA ribosomal RNA gene database project: improved data processing and web-based tools. Nucleic Acids Research 41:D590-D596. DOI: 10.1093/nar/gks1219. 
484 Round JL, Mazmanian SK. 2009. The gut microbiota shapes intestinal immune responses during

485

486

487

488

489

490

491

492

493

494

495

496

497

498

499

500

501

502

503

504

505

506

507

508

509 health and disease. Nature reviews immunology 9:313-323.

Schokker D, Zhang J, Zhang L, Vastenhouw SA, Heilig HG, Smidt H, Rebel JM, Smits MA. 2014. Early-life environmental variation affects intestinal microbiota and immune development in new-born piglets. PloS one 9:e100040.

Slifierz MJ, Friendship RM, Weese JS. 2015. Longitudinal study of the early-life fecal and nasal microbiotas of the domestic pig. BMC microbiology 15:1-12.

Stalder KJ, Lacy RC, Cross TL, Conatser GE. 2003. Financial impact of average parity of culled females in a breed-to-wean swine operation using replacement gilt net present value analysis. Journal of Swine Health and Production 11:69-74.

Stein TE, Dijkhuizen A, D’Allaire S, Morris RS. 1990. Sow culling and mortality in commercial swine breeding herds. Preventive Veterinary Medicine 9:85-94.

Sutherland D.The Marketing Journey of Cull Sows and Secondary Market Pigs - Swine Health Information Center. 2018. Available at https://www.swinehealth.org/the-marketingjourney/ (accessed February 11, 2021).

Weese JS, Slifierz M, Jalali M, Friendship R. 2014. Evaluation of the nasal microbiota in slaughter-age pigs and the impact on nasal methicillin-resistant Staphylococcus aureus (MRSA) carriage. BMC veterinary research 10:1-10.

Yilmaz P, Parfrey LW, Yarza P, Gerken J, Pruesse E, Quast C, Schweer T, Peplies J, Ludwig W, Glöckner FO. 2014. The SILVA and "All-species Living Tree Project (LTP)" taxonomic frameworks. Nucleic Acids Research 42:D643-D648. DOI: 10.1093/nar/gkt1209.

Young VB. 2017. The role of the microbiome in human health and disease: an introduction for clinicians. Bmj 356. 
510 Figure titles and legends:

511

512 Figure 1

513 Title: Sampling protocol with summarized sample processing steps and relative locations of the

514 six participating farms. Numbers represent farms 1 to 6 . Actual map is not shown for

515 confidentiality reasons.

516

517 Figure 2

518 Title: Alpha diversity by farm and health status. Microbial diversity (Shannon index) did not

519 differ by farm or health status for pooled fecal samples and pooled nasal swabs. Median and

520 quartiles are shown in the box and whiskers plots. Fecal samples were rarefied at 5600 reads and

521 nasal samples were rarefied at 500 reads.

522

523

Figure 3

524 Title: Nasal microbial communities (Bray Curtis) by a) farm and b) health status.

525 Legend: Nasal samples were rarefied to 500 reads and differed significantly by farm

526 (PERMANOVA $\mathrm{p}=0.002$ ) and by health status (PERMANOVA $\mathrm{p}=0.017$ ). No pairwise

527 comparisons were significant by farm. Healthy versus cull was significant $(\mathrm{p}=0.033)$ by health

528 status.

529

530 Figure 4

531 Title: Fecal microbial communities (Bray Curtis) by a) farm and b) health status

532 Legend: Fecal samples were rarefied to 5600 reads and differed significantly by farm

533 (PERMANOVA $\mathrm{p}=0.001$ ) but not by health status (PERMANOVA $\mathrm{p}=0.663$ ). Pairwise

534 comparisons were significant between all farms $(\mathrm{p}<0.05)$ with the exception of farms 4 and $5(\mathrm{p}$

$535=0.08)$ and farms 5 and $6(\mathrm{p}=0.1)$. No significant differences were found based on health status

$536(\mathrm{p}=0.663)$.

537

538 Figure 5

539 Title: Microbial taxa bar plots at the phyla level in a) nasal samples and b) fecal samples. Farm

540 and health status of each sample is identified. All taxa with $>1 \%$ abundance are included.

541

542 Supplemental Figure 1

543 Title: Alpha diversity analysis (Faith PD index) for pooled fecal and nasal swabs.

544 Legend: Microbial diversity by a) sample type and b) farm and health status. In A, all nasal and

545 fecal samples were rarefied at 500 reads. In B, fecal samples were rarefied at 5600 reads and

546 nasal samples were rarefied at 500 reads. Median and quartiles are shown in the box and

547 whiskers plots. $\mathrm{N}=36$ fecal and 19 nasal swabs. ${ }^{*} \mathrm{p}<0.001$

548

549 Supplemental Figure 2 
550 Title: Nasal microbial communities (Jacaard) by a) farm and b) health status.

551

552 Supplemental Figure 3

553 Title: Fecal microbial communities (Jacaard) by a) farm and b) health status.

554

555 Supplemental Table 1

556 Title: Number of reads in each sample. (Chloroplasts, mitochondria, and eukaryotic reads have

557 been removed.)

558

559 Supplemental Table 2

560 Title: Nasal samples - Average number of reads by Farm and Health Status. (Samples with fewer

561 than 500 reads were excluded.)

562

563 Supplemental Table 3

564 Title: Fecal samples - average number of reads by Farm and Health Status. (Samples with fewer

565 than 5600 reads were excluded.)

566

567 Supplemental Table 4

568 Title: Microbial community analysis of nasal samples with and without rarefaction at 500 reads

569 and 1000 reads. Microbial community analysis of fecal samples with and without rarefaction at

5705600 reads.

571

572 Supplemental Table 5

573 Title: L7 feature table for pooled fecal samples

574

575 Supplemental Table 6

576 Title: L7 feature table for pooled nasal swab samples

577 


\section{Table 1 (on next page)}

Farm demographics and general information for the source population of the study

Low performance included notes that has any of the following parameters: low born alive, low number of weaned pigs, low milk production, and low performance. 
1 Table 1. Farm demographics and general information for the source population of the study

\begin{tabular}{|c|c|c|c|c|c|c|}
\hline Descriptor & Farm 1 & Farm 2 & Farm 3 & Farm 4 & Farm 5 & Farm 6 \\
\hline Date sampled & $6 / 26 / 2017$ & $7 / 10 / 2017$ & $7 / 10 / 2017$ & $7 / 12 / 2017$ & $7 / 12 / 2017$ & $7 / 18 / 2017$ \\
\hline $\begin{array}{l}\text { Number of } \\
\text { sows }\end{array}$ & 1,300 & 2,700 & 1,100 & 2,400 & 5,500 & 4,000 \\
\hline $\begin{array}{l}\text { Cull sow } \\
\text { housing type }\end{array}$ & Crates & Crates & Crates & Crates/ Pens & $\begin{array}{l}\text { Crates/ } \\
\text { Pens }\end{array}$ & Crates \\
\hline $\begin{array}{l}\text { Cull sow } \\
\text { removal } \\
\text { frequency }\end{array}$ & \begin{tabular}{|l} 
Every 2 \\
weeks
\end{tabular} & $\begin{array}{l}\text { Every 1-2 } \\
\text { weeks }\end{array}$ & $\begin{array}{l}\text { Every } 2 \\
\text { weeks }\end{array}$ & $\begin{array}{l}\text { Every } 2 \\
\text { weeks }\end{array}$ & Weekly & $\begin{array}{l}\text { Every } 2 \\
\text { weeks }\end{array}$ \\
\hline $\begin{array}{l}\text { Mean (SD) } \\
\text { parity }\end{array}$ & $3.4(1.6)$ & $3.5(2.2)$ & $3.3(1.8)$ & $3.9(2.2)$ & $4.3(3.0)$ & $3.4(1.8)$ \\
\hline $\begin{array}{l}\text { Top three } \\
\text { listed reasons } \\
\text { for } \\
\text { 'compromised } \\
\text { / clinical' } \\
\text { sows }(\%)\end{array}$ & $\begin{array}{l}\text { Low body } \\
\text { condition } \\
(50), \\
\text { abortion/ } \\
\text { discharge } \\
(40), \\
\text { lameness } \\
(10)\end{array}$ & $\begin{array}{l}\text { Low body } \\
\text { condition } \\
\text { (40), } \\
\text { abortion/ } \\
\text { discharge } \\
\text { (30), } \\
\text { shoulder } \\
\text { ulcer, } \\
\text { abcess, } \\
\text { other/ } \\
\text { kissing (10 } \\
\text { for each) }\end{array}$ & $\begin{array}{l}\text { Low body } \\
\text { condition } \\
(40), \\
\text { shoulder } \\
\text { ulcer }(20) \text {, } \\
\text { other/ } \\
\text { missing } \\
(20)\end{array}$ & $\begin{array}{l}\text { Low body } \\
\text { condition } \\
(50), \\
\text { abortion/ } \\
\text { discharge } \\
(30) \text {, } \\
\text { lameness } \\
\text { and } \\
\text { shoulder } \\
\text { ulcer (10 } \\
\text { each) }\end{array}$ & $\begin{array}{l}\text { Low body } \\
\text { condition } \\
(70), \\
\text { abortion/ } \\
\text { discharge } \\
(20), \\
\text { shoulder } \\
\text { ulcer (10) }\end{array}$ & $\begin{array}{l}\text { Low body } \\
\text { condition } \\
\text { and } \\
\text { shoulder } \\
\text { ulcer ( } 40 \\
\text { each), } \\
\text { abortion/ } \\
\text { discharge } \\
\text { (20) }\end{array}$ \\
\hline $\begin{array}{l}\text { Top three } \\
\text { listed reasons } \\
\text { for 'cull' sows } \\
(\%)\end{array}$ & \begin{tabular}{|l|} 
Low \\
performanc \\
e (70), \\
return to \\
estrus (30)
\end{tabular} & $\begin{array}{l}\text { Low } \\
\text { performanc } \\
\text { e (40), } \\
\text { return to } \\
\text { estrus (30), } \\
\text { missing } \\
\text { information } \\
(30)\end{array}$ & $\begin{array}{l}\text { High parity } \\
(40), \text { low } \\
\text { performanc } \\
\text { e (30), } \\
\text { missing } \\
\text { information } \\
(30)\end{array}$ & $\begin{array}{l}\text { Low } \\
\text { performanc } \\
\text { e }(100)\end{array}$ & $\begin{array}{l}\text { Missing } \\
\text { informatio } \\
\mathrm{n}(100)\end{array}$ & $\begin{array}{l}\text { Low } \\
\text { performanc } \\
\text { e (80), } \\
\text { missing } \\
\text { information } \\
(20)\end{array}$ \\
\hline
\end{tabular}

2 Low performance included notes that has any of the following parameters: low born alive, low

3 number of weaned pigs, low milk production, and low performance. 
Figure 1

Sampling protocol with summarized sample processing steps (A) and relative locations (B) of the six participating farms.

Numbers represent farms 1 to 6 . Actual map is not shown for confidentiality reasons. 


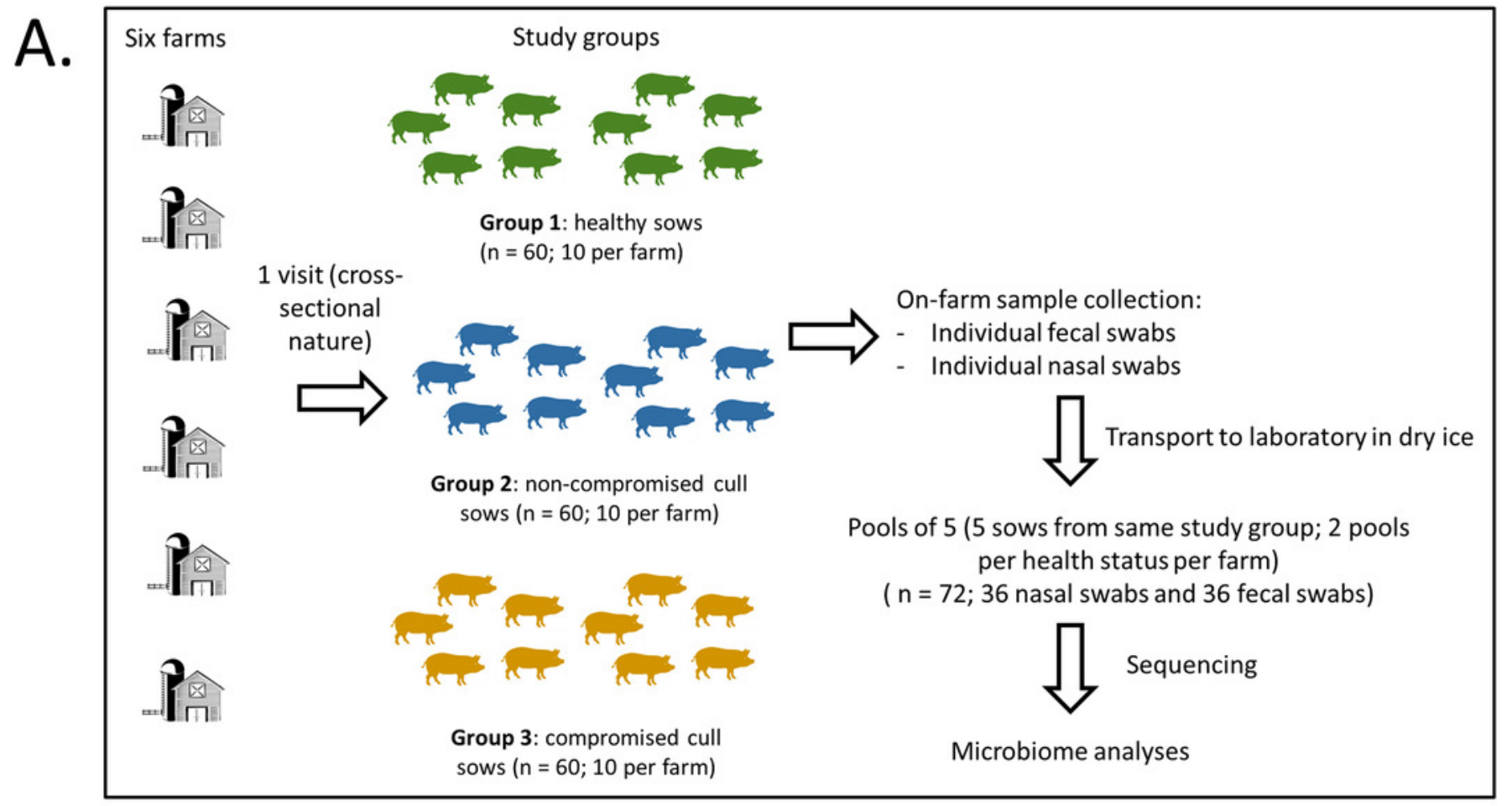

B.

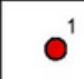

$0^{2}$

$0^{3}$

$0^{4}$ 
Figure 2

Alpha diversity by farm and health status.

Microbial diversity (Shannon index) did not differ by farm or health status for pooled fecal samples and pooled nasal swabs. Median and quartiles are shown in the box and whiskers plots. Fecal samples were rarefied at 5600 reads and nasal samples were rarefied at 500 reads.

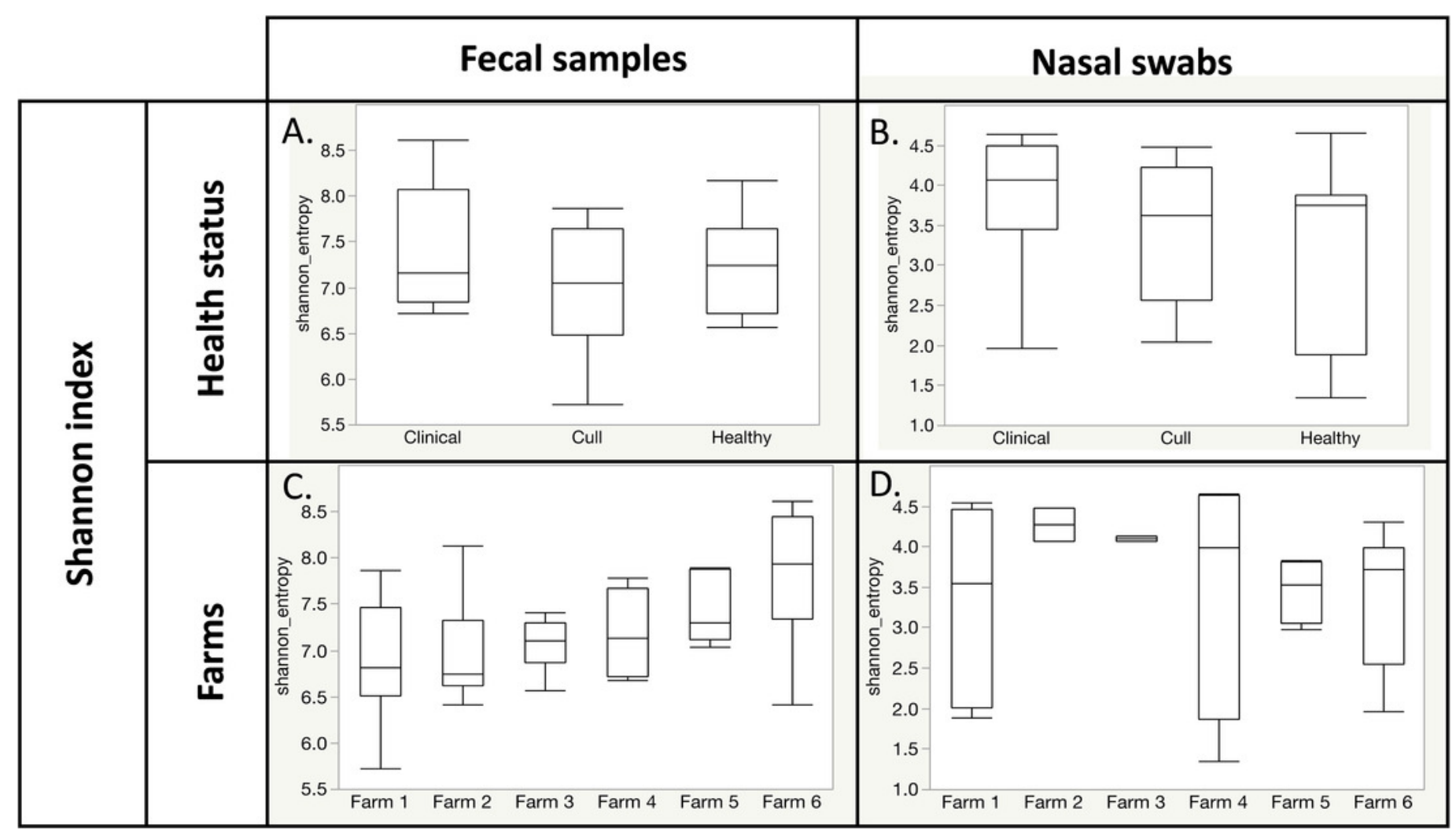


Figure 3

Nasal microbial communities (Bray Curtis) by a) farm and b) health status.

Nasal samples were rarefied to 500 reads and differed significantly by farm (PERMANOVA $p=$ 0.002 ) and by health status (PERMANOVA $p=0.017$ ). No pairwise comparisons were significant by farm. Healthy versus cull was significant $(p=0.033)$ by health status.
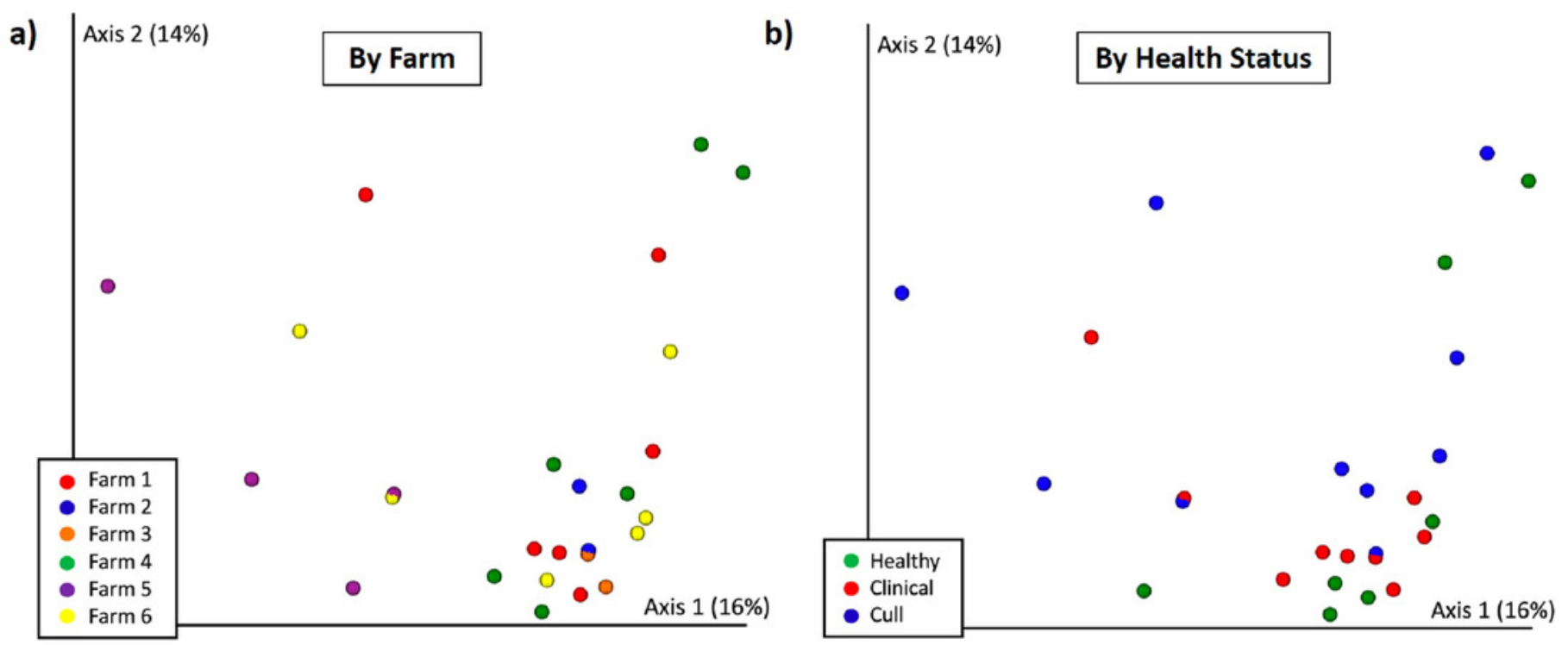
Figure 4

Fecal microbial communities (Bray Curtis) by a) farm and b) health status

Fecal samples were rarefied to 5600 reads and differed significantly by farm (PERMANOVA $p$ $=0.001$ ) but not by health status (PERMANOVA $p=0.663$ ). Pairwise comparisons were significant between all farms $(p<0.05)$ with the exception of farms 4 and $5(p=0.08)$ and farms 5 and $6(p=0.1)$. No significant differences were found based on health status $(p=$ $0.663)$.

A.

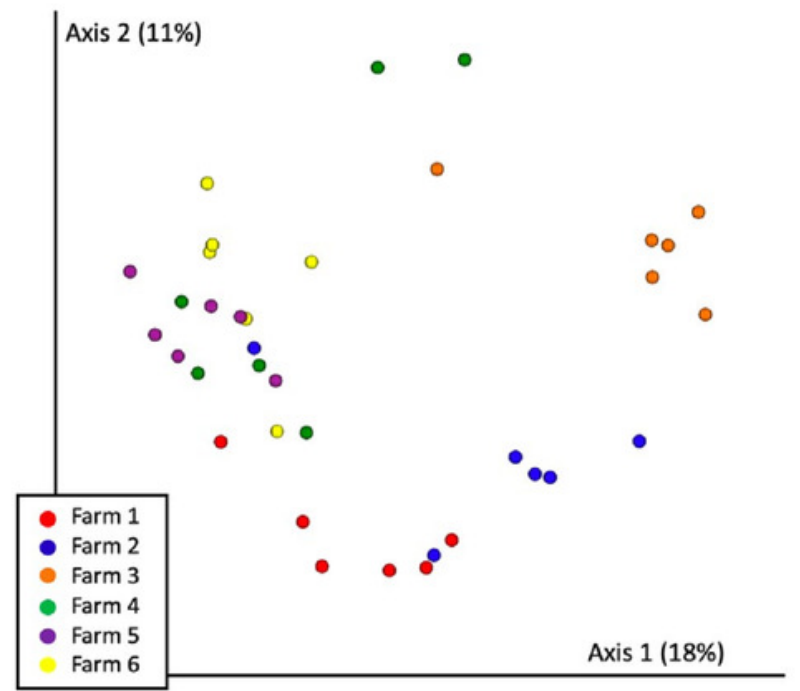

B.

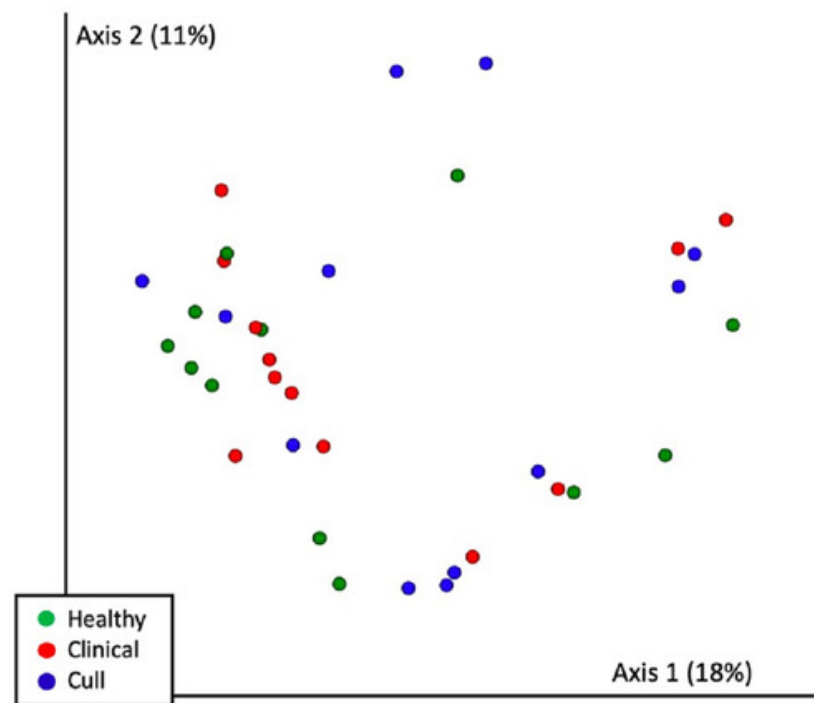




\section{Figure 5}

Microbial taxa bar plots at the phyla level in a) nasal samples and b) fecal samples.

Farm and health status of each sample is identified. All taxa with $>1 \%$ abundance are included.
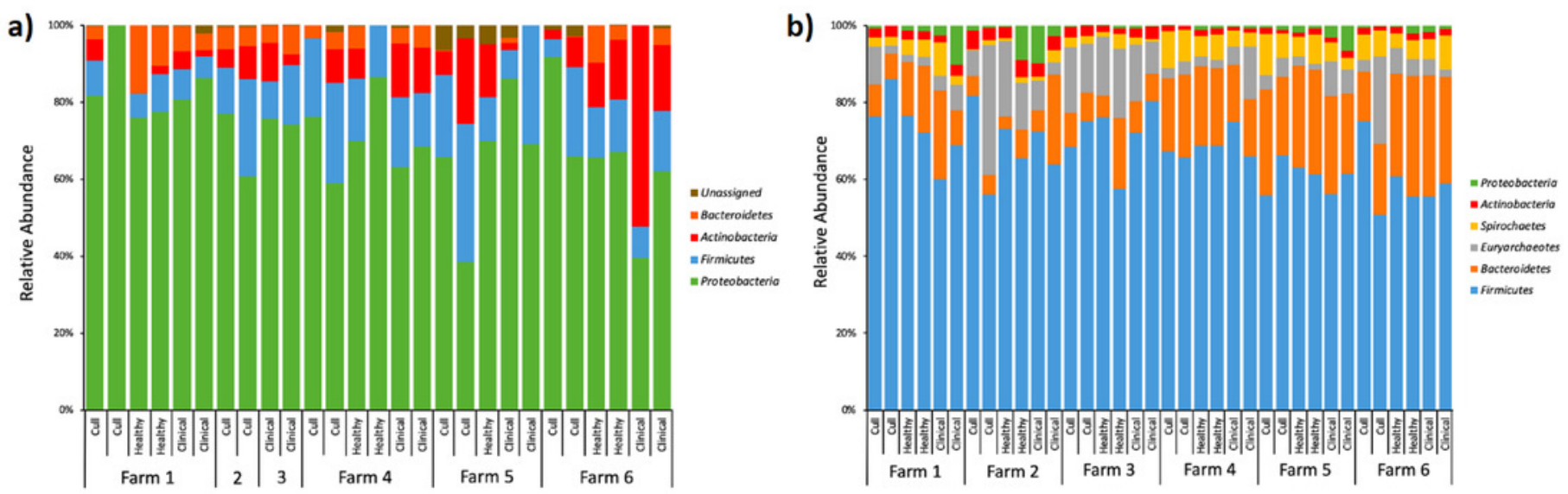